\title{
Elevated Midtrimester Triglycerides as a Biomarker for Postpartum Hyperglycemia in Gestational Diabetes
}

\author{
Mengyu Lai $\left(\mathbb{D},{ }^{1}\right.$ Fang Fang, ${ }^{1}$ Yuhang Ma, ${ }^{1}$ Jiaying Yang, ${ }^{1}$ Jingjing Huang, ${ }^{1} \mathrm{Na} \mathrm{Li},{ }^{1}$ Mei Kang,

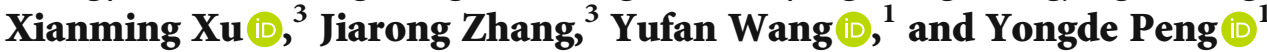 \\ ${ }^{1}$ Department of Endocrinology and Metabolism, Shanghai General Hospital, Shanghai Jiao Tong University, Shanghai, China \\ ${ }^{2}$ Clinical Research Center, Shanghai General Hospital, Shanghai Jiao Tong University, Shanghai, China \\ ${ }^{3}$ Department of Obstetrics and Gynecology, Shanghai General Hospital, Shanghai Jiao Tong University, Shanghai, China
}

Correspondence should be addressed to Yufan Wang; yyffwang@sina.com

Received 13 January 2020; Revised 9 March 2020; Accepted 3 April 2020; Published 24 April 2020

Academic Editor: Eric Hajduch

Copyright (c) 2020 Mengyu Lai et al. This is an open access article distributed under the Creative Commons Attribution License, which permits unrestricted use, distribution, and reproduction in any medium, provided the original work is properly cited.

\begin{abstract}
Background. Whether elevated triglyceride (TG) levels during pregnancy were a biomarker for postpartum abnormal glucose metabolism (AGM) in women with previous gestational diabetes mellitus (GDM) remained unknown. The aim of this study was to investigate the association between TG levels during the second trimester and postpartum AGM in GDM women. Methods. This was a retrospective cohort study including 513 GDM women. A $75 \mathrm{~g}$ oral glucose tolerance test (OGTT) was performed, and lipid levels were determined during pregnancy and the postpartum period. GDM patients were categorized into tertiles according to their TG levels at $24-28$ weeks of gestation (TG $<2.14 \mathrm{mmol} / \mathrm{L}$, TG: $2.14-2.89 \mathrm{mmol} / \mathrm{L}$, and TG $>2.89$ $\mathrm{mmol} / \mathrm{L}$ ). A logistic regression model was used to calculate the odds ratios (ORs) and 95\% confidence intervals (CIs). Results. During pregnancy, women in the high TG tertile showed higher HbAlc levels $(5.47 \pm 0.58 \%$ versus $5.28 \pm 0.49 \%, p=0.006)$, higher total cholesterol (TC) levels $(5.85 \pm 1.23 \mathrm{mmol} / \mathrm{L}$ versus $5.15 \pm 0.97 \mathrm{mmol} / \mathrm{L}, p=0.026)$, and higher HOMA-IR (2.36 $(1.62-3.45)$ versus $1.49(0.97-2.33), p<0.001)$ than the participants in the low TG tertile. After delivery, the prevalence rates of AGM based on above tertiles of TG levels during pregnancy were $26.90 \%, 33.33 \%$, and $43.27 \%$, respectively $(p=0.006)$. High TG tertile during the second trimester was associated with the presence of postpartum AGM (adjusted OR: 2.001, 95\% CI: 1.054-3.800, $p=0.034)$. Conclusions. The elevated midtrimester TG levels were not only accompanied by higher glucose and lipid levels and more severe insulin resistance at the time of the measurement but were a biomarker for postpartum AGM as well.
\end{abstract}

\section{Introduction}

Gestational diabetes mellitus (GDM) is a pregnancy-related metabolic disorder, which affects $4-18 \%$ of pregnant women in various countries [1]. GDM not only increases the risk of maternal and fetal perinatal complications in the short term but also increases the risk of subsequently developing type 2 diabetes mellitus (T2DM) and cardiovascular disease in the long term [2-5]. The American Diabetes Association (ADA) recommends performing a $75 \mathrm{~g}$ oral glucose tolerance test (OGTT) over hemoglobin Alc (HbAlc) at 4-12 weeks postpartum for every GDM patient to evaluate her glucose metabolism [6,7]. Intensified surveillance, early detection of risk factors, and active intervention may reduce postpartum abnormal glucose metabolism (AGM).

An elevated triglyceride (TG) profile is a common lipid abnormality that accompanies T2DM and prediabetic states [8]. Hyperlipidemia is more severe in pregnant women with GDM than in normal glucose pregnant women [9]. Studies indicated that maternal lipid, which changed throughout gestation to meet the needs of fetal growth, played an important role on obstetric complications [10]. Further, prospective studies showed that the TG level was an independent risk factor for developing diabetes in middle-aged women $[11,12]$. In a prospective case-control study, higher TG levels were observed at 11-14 weeks of gestation in women with GDM, 


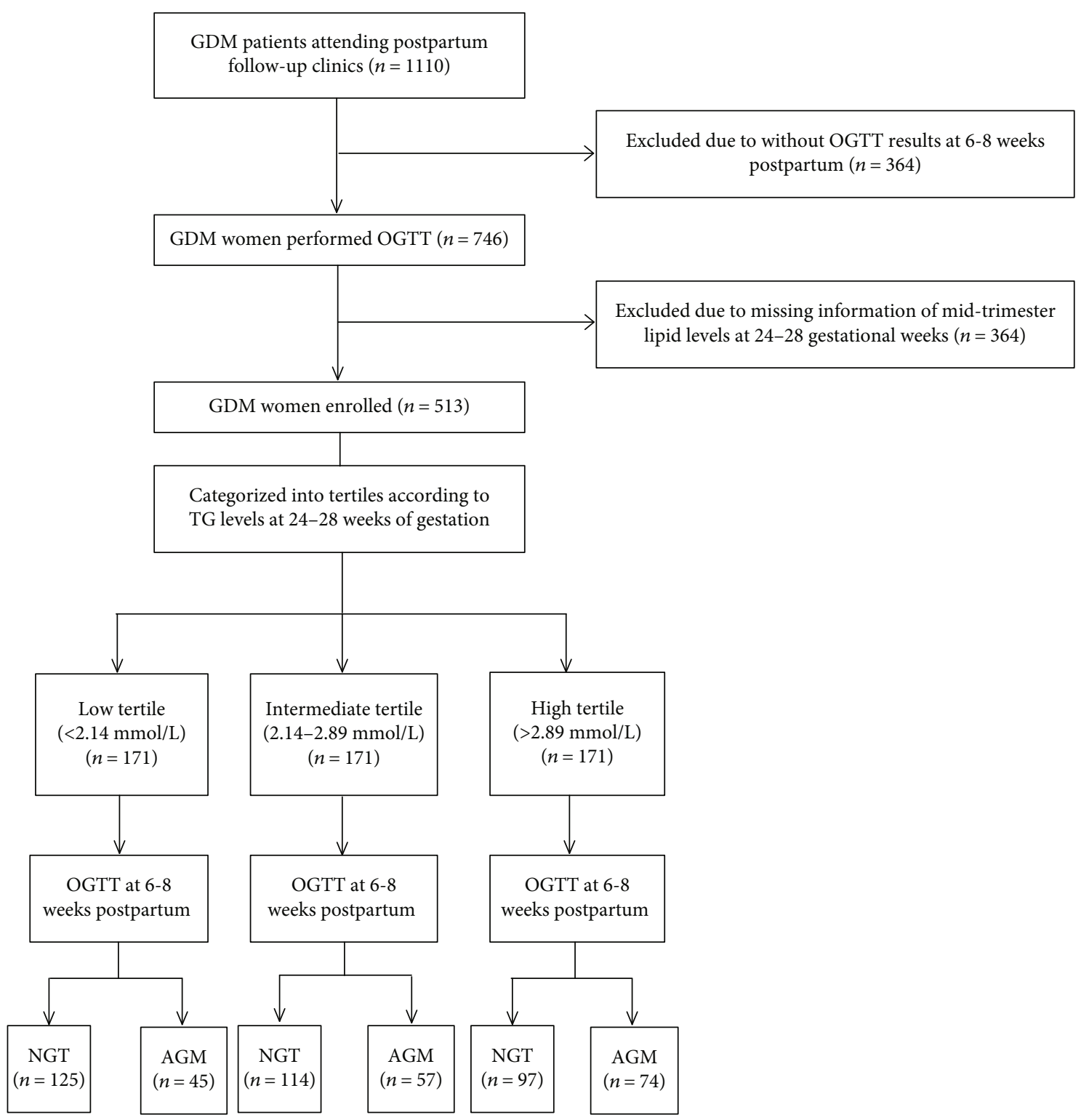

FIGURE 1: Subject screening and distribution. GDM: gestational diabetes mellitus; OGTT: oral glucose tolerance test; TG: triglyceride; NGT: normal glucose tolerance; AGM: abnormal glucose metabolism.

and multivariate regression analysis indicated that TG levels were a significant predictor of a subsequent diagnosis of GDM [13]. However, fewer studies have characterized the relationship between TG levels during pregnancy and the risk of postpartum AGM in GDM patients.

Here, we explored the relationship between TG levels during midterm pregnancy and postpartum glucose metabolism in Chinese women with previous GDM. We aimed to determine whether midterm elevated TG levels were a biomarker for the development of sustained hyperglycemia after delivery.

\section{Materials and Methods}

2.1. Subjects. This was a retrospective cohort study with 1110 GDM patients attending the postpartum follow-up clinics in
Shanghai General Hospital between April 2012 and April 2019. In the present analysis, we excluded 364 women who did not attend the OGTT at 6-8 weeks postpartum and 233 women who did not test midtrimester lipid levels. The remaining 513 GDM women were included in the final analysis (Figure 1). Each enrolled pregnant woman was administered a $75 \mathrm{~g}$ OGTT at 24-28 weeks of gestation and 6-12 weeks after delivery. In addition, fasting lipid levels, including serum total cholesterol (TC), TGs, high-density lipoproteincholesterol (HDL-C), and low-density lipoprotein-cholesterol (LDL-C), insulin levels, alanine aminotransferase (ALT), and aspartate aminotransferase (AST) were detected. Women were diagnosed with GDM if one or more plasma glucose values during the $75 \mathrm{~g}$ OGTT at 24-28 gestational weeks were met or exceeded: $0 \mathrm{~h}, 5.1 \mathrm{mmol} / \mathrm{L} ; 1 \mathrm{~h}, 10.0 \mathrm{mmol} / \mathrm{L} ;$ and $2 \mathrm{~h}$, $8.5 \mathrm{mmol} / \mathrm{L}$ [14]. The subjects underwent a $75 \mathrm{~g}$ OGTT at $6-8$ 
weeks after delivery, and the diagnosis of normal glucose tolerance (NGT), T2DM, impaired fasting glucose (IFG), and impaired glucose tolerance (IGT) was based on the World Health Organization (WHO) diagnostic criteria published in 1999 [15]. According to TG levels after delivery, the patients were diagnosed with normal TG (NTG, TG $<1.70 \mathrm{mmol} / \mathrm{L}$ ) and abnormal TG (ATG, TG $\geq 1.70 \mathrm{mmol} / \mathrm{L}$ ) [16]. Each patient provided informed consent, and the research was carried out in compliance with the Declaration of Helsinki. The study protocol was approved by the ethics committee of Shanghai General Hospital, Shanghai Jiao Tong University.

2.1.1. Grouping. (1) All GDM patients were categorized into tertiles according to their TG levels at 24-28 weeks of gestation as follows: low tertile $(<2.14 \mathrm{mmol} / \mathrm{L})$, intermediate tertile $(2.14-2.89 \mathrm{mmol} / \mathrm{L})$, and high tertile $(>2.89 \mathrm{mmol} / \mathrm{L})$. (2) The patients were also categorized into two groups according to the results of the postpartum OGTT: the NGT group (fasting plasma glucose (FPG) $<6.1 \mathrm{mmol} / \mathrm{L}$ and $2 \mathrm{~h}$ glucose load plasma glucose $(2 \mathrm{hPG})<7.8 \mathrm{mmol} / \mathrm{L})$ and the AGM group (FPG $\geq 6.1 \mathrm{mmol} / \mathrm{L}$ or $2 \mathrm{hPG} \geq 7.8 \mathrm{mmol} / \mathrm{L}$ ) (Figure 1).

2.2. Data Collection. The following maternal characteristics were assessed: age at delivery, height, body weight before pregnancy and at 6-8 weeks postpartum, first-degree family history of diabetes, and insulin treatment during pregnancy.

2.3. Metabolic Measurements. Plasma glucose levels were measured enzymatically. Fasting insulin (FINS) and serum lipid (TC, TG, HDL-C, and LDL-C) levels were determined by chemiluminescent assays. ALT and AST levels were determined by enzymatic methods. HbAlc and glycated albumin (GA) levels were determined by high-performance liquid chromatography. HOMA- $\beta$ and HOMA-IR were calculated to evaluate $\beta$ cell function and insulin resistance using the following formulas: HOMA- $\beta=20 \times$ FINS $(\mu \mathrm{U} / \mathrm{mL}) /(\mathrm{FPG}$ $(\mathrm{mmol} / \mathrm{L})-3.5)$ and HOMA-IR $=$ FPG $(\mathrm{mmol} / \mathrm{L}) \times$ FINS $(\mu \mathrm{U} / \mathrm{mL}) / 22.5 \quad[17]$.

2.4. Statistical Analysis. Data were expressed as the mean \pm standard deviation for normally distributed variables and as the median with the interquartile range for skewed data. To determine the differences among the three groups, we conducted analysis of variance or the Kruskal-Wallis test for continuous variables and a chi-square test for categorical variables. Least significance difference tests were used to perform pairwise comparisons between two groups. Logistic regression analysis was used to determine whether elevated TG levels during gestation were independently associated with AGM. Statistical significance was set at $p<0.05$. Statistical analyses were carried out using SPSS (IBM Corp., Armonk, NY).

\section{Results}

3.1. Demographic and Metabolic Characteristics of GDM Patients at 24-28 Gestational Weeks, Categorized into Tertiles according to TG Levels at the Time of Measurement. There were significant differences among the three groups concerning metabolic levels during pregnancy. The partici- pants in the high TG tertile had a higher prepregnancy BMI $\left(23.74 \pm 3.47 \mathrm{~kg} / \mathrm{m}^{2}\right.$ versus $22.12 \pm 3.54 \mathrm{~kg} / \mathrm{m}^{2}, p<$ $0.001)$, higher FPG levels $(5.25 \pm 0.97 \mathrm{mmol} / \mathrm{L}$ versus $4.82 \pm 0.80 \mathrm{mmol} / \mathrm{L}, p<0.001)$, higher $1 \mathrm{~h}$ plasma glucose (1hPG) levels $(10.58 \pm 1.94 \mathrm{mmol} / \mathrm{L}$ versus $9.73 \pm 1.86$ $\mathrm{mmol} / \mathrm{L}, p<0.001)$, higher $2 \mathrm{~h}$ plasma glucose $(2 \mathrm{hPG})$ levels $(9.13 \pm 2.03 \mathrm{mmol} / \mathrm{L}$ versus $8.29 \pm 1.81 \mathrm{mmol} / \mathrm{L}, p<$ $0.001)$, higher $\mathrm{HbAlc}(5.47 \pm 0.58 \%$ versus $5.28 \pm 0.49 \%$, $p=0.006)$, higher TC levels $(5.85 \pm 1.23 \mathrm{mmol} / \mathrm{L}$ versus $5.15 \pm 0.97 \mathrm{mmol} / \mathrm{L}, \quad p=0.026)$, lower HDL-C levels $(1.64 \pm 0.34 \mathrm{mmol} / \mathrm{L}$ versus $1.88 \pm 0.47 \mathrm{mmol} / \mathrm{L}, p<0.001)$ and higher HOMA-IR (2.36 (1.62-3.45) versus 1.49 (0.97-2.33), $p<0.001)$ than the participants in the low TG tertile (Table 1).

3.2. Glucose and Lipid Levels of GDM Patients at 6-8 Weeks Postpartum. After delivery, approximately 34.5\% (177/513) of the GDM patients had sustained high glucose levels. The rates of IFG, IGT, IFG combined with IGT, and DM were $1.2 \%$ (6/513), $24.0 \%$ (123/513), 2.7\% (14/513), and 6.6\% (34/513), respectively. The TG levels of most GDM patients returned to normal 6-8 weeks after delivery; however, $25.0 \%(128 / 513)$ of the women continued to have hypertriglyceridemia.

3.3. Demographic and Metabolic Characteristics of GDM Patients at 6-8 Weeks Postpartum Categorized into Tertiles according to TG Levels at 24-28 Gestational Weeks. There were significant differences among the three groups concerning metabolic levels after delivery. The participants in the high TG tertile showed a higher postpartum BMI $\left(25.03 \pm 3.14 \mathrm{~kg} / \mathrm{m}^{2}\right.$ versus $23.56 \pm 3.30 \mathrm{~kg} / \mathrm{m}^{2}, p<0.001$ ), higher FPG levels $(5.18 \pm 0.90 \mathrm{mmol} / \mathrm{L}$ versus $4.87 \pm 0.68 \mathrm{mmol} / \mathrm{L}, p=0.002)$, higher $1 \mathrm{hPG}$ levels $(9.66 \pm 2.43 \mathrm{mmol} / \mathrm{L}$ versus $9.11 \pm 2.15$ $\mathrm{mmol} / \mathrm{L}, p=0.048)$, higher $2 \mathrm{hPG}$ levels $(7.64 \pm 2.36 \mathrm{mmol} / \mathrm{L}$ versus $6.99 \pm 1.98 \mathrm{mmol} / \mathrm{L}, \quad p=0.022)$, higher TC levels $(5.36 \pm 0.91 \mathrm{mmol} / \mathrm{L}$ versus $5.09 \pm 0.99 \mathrm{mmol} / \mathrm{L}, p=0.026)$, higher LDL-C levels $(3.39 \pm 0.73 \mathrm{mmol} / \mathrm{L}$ versus $3.04 \pm 0.92$ $\mathrm{mmol} / \mathrm{L}, p=0.001)$, lower HDL-C levels $(1.34 \pm 0.85 \mathrm{mmol} / \mathrm{L}$ versus $1.54 \pm 0.32 \mathrm{mmol} / \mathrm{L}, p<0.001)$ and higher HOMA-IR (1.59 (0.97-2.43) versus $1.10(0.69-1.63), p<0.001)$ than the participants in the low TG tertile. In addition, the prevalence rates of AGM were $43.27 \%, 33.33 \%$, and $26.90 \%(p=0.005)$ in the three groups (TG $>2.89 \mathrm{mmol} / \mathrm{L}$, TG: $2.14-2.89 \mathrm{mmol} / \mathrm{L}$, and $\mathrm{TG}<2.14 \mathrm{mmol} / \mathrm{L}$ ), respectively (Table 2 ).

3.4. Elevated TG Levels as a Biomarker for the Development of Postpartum AGM in GDM Patients. To determine whether the elevated TG level during the second trimester of pregnancy was a biomarker for postpartum AGM, the odds ratios (ORs) for AGM in women with different TG levels during the second trimester were calculated. First, compared to the low TG tertile group at 24-28 gestational weeks, the crude OR for AGM was 2.073 (95\% CI 1.317-3.263, $p=0.002$ ) for the high TG tertile group, and the adjusted OR was 2.001 (95\% CI $1.054-3.800, p=0.034$ ) when considering age, prepregnancy $\mathrm{BMI}$, family DM history, insulin treatment during pregnancy, FPG during the second trimester, $\mathrm{HbAlc}$ during the second trimester, macrosomia, ALT, and AST (Table 3). In 
TABLE 1: Demographic and metabolic characteristics of GDM patients at 24-28 gestational weeks, categorized into tertiles according to TG levels at the time of measurement.

\begin{tabular}{|c|c|c|c|c|}
\hline Variables & $\begin{array}{c}\text { Low tertile } \\
(<2.14 \mathrm{mmol} / \mathrm{L}) \\
(N=171) \\
\end{array}$ & $\begin{array}{c}\text { Intermediate tertile } \\
(2.14 \sim 2.89 \mathrm{mmol} / \mathrm{L}) \\
(N=171)\end{array}$ & $\begin{array}{c}\text { High tertile } \\
(>2.89 \mathrm{mmol} / \mathrm{L}) \\
(N=171) \\
\end{array}$ & $p$ value \\
\hline Prepregnancy BMI $\left(\mathrm{kg} / \mathrm{m}^{2}\right)$ & $22.12 \pm 3.54$ & $22.78 \pm 3.21^{*}$ & $23.74 \pm 3.47^{* *}$ & 0.0001 \\
\hline $\mathrm{FPG}(\mathrm{mmol} / \mathrm{L})$ & $4.82 \pm 0.80$ & $4.90 \pm 0.79$ & $5.25 \pm 0.97^{* *}$ & 0.0001 \\
\hline $1 \mathrm{hPG}(\mathrm{mmol} / \mathrm{L})$ & $9.73 \pm 1.86$ & $10.23 \pm 1.67$ & $10.58 \pm 1.94^{* *}$ & 0.0001 \\
\hline 2hPG (mmol/L) & $8.29 \pm 1.81$ & $8.68 \pm 1.84^{* *}$ & $9.13 \pm 2.03^{* *}$ & 0.0001 \\
\hline HbAlc (\%) & $5.28 \pm 0.49$ & $5.33 \pm 0.48$ & $5.47 \pm 0.58^{* *}$ & 0.006 \\
\hline GA (\%) & $13.60 \pm 1.76$ & $13.46 \pm 2.00$ & $13.28 \pm 2.09$ & 0.396 \\
\hline $\mathrm{TC}(\mathrm{mmol} / \mathrm{L})$ & $5.15 \pm 0.97$ & $5.75 \pm 1.04$ & $5.85 \pm 1.23^{*}$ & 0.016 \\
\hline HDL-C (mmol/L) & $1.88 \pm 0.47$ & $1.80 \pm 0.34$ & $1.64 \pm 0.34^{* *}$ & 0.0001 \\
\hline LDL-C (mmol/L) & $3.12 \pm 0.72$ & $3.25 \pm 0.80$ & $3.17 \pm 0.98$ & 0.356 \\
\hline $\operatorname{ALT}(\mathrm{U} / \mathrm{L})$ & $12.20(9.43-18.08)$ & $12.15(9.30-19.75)$ & $10.90(9.05-16.60)$ & 0.574 \\
\hline AST (U/L) & $16.10(13.23-19.78)$ & $16.05(13.60-20.78)$ & $15.40(12.50-19.90)$ & 0.483 \\
\hline HOMA- $\beta(\%)$ & $123.87(87.15-167.03)$ & 139.73 (91.90-198.51) & $124.10(91.10-179.72)$ & 0.290 \\
\hline HOMA-IR & $1.49(0.97-2.33)$ & $1.89(1.31-2.75)^{* *}$ & $2.36(1.62-3.45)^{* *}$ & 0.0001 \\
\hline
\end{tabular}

Data are expressed as the mean \pm SD or median (interquartile range). TG: triglyceride; BMI: body mass index; FPG: fasting plasma glucose; $1 \mathrm{hPG:} 1 \mathrm{~h}$ plasma glucose; 2hPG: 2 h plasma glucose; HbAlc: hemoglobin Alc; GA: glycated albumin; TC: total cholesterol; HDL-C: high-density lipoprotein-cholesterol; LDL-C: low-density lipoprotein-cholesterol; ALT: alanine aminotransferase; AST: aspartate aminotransferase; HOMA-IR: homeostasis model assessment for insulin resistance index; HOMA- $\beta$ : homeostasis model assessment for $\beta$ cell function. ${ }^{*}$ Compared to the low tertile group, $p<0.05 .{ }^{* *}$ Compared to the low tertile group, $p<0.01$.

TABle 2: Demographic and metabolic characteristics of GDM patients at 6-8 weeks postpartum categorized into tertiles according to TG levels at 24-28 gestational weeks.

\begin{tabular}{|c|c|c|c|c|}
\hline Variables & $\begin{array}{c}\text { Low tertile } \\
(<2.14 \mathrm{mmol} / \mathrm{L}) \\
(N=171)\end{array}$ & $\begin{array}{c}\text { Intermediate tertile } \\
(2.14 \sim 2.89 \mathrm{mmol} / \mathrm{L}) \\
\quad(N=171)\end{array}$ & $\begin{array}{c}\text { High tertile } \\
(>2.89 \mathrm{mmol} / \mathrm{L}) \\
(N=171)\end{array}$ & $p$ value \\
\hline Age (years) & $31.49 \pm 4.45$ & $32.07 \pm 4.90$ & $32.29 \pm 4.07$ & 0.140 \\
\hline Postpartum BMI $\left(\mathrm{kg} / \mathrm{m}^{2}\right)$ & $23.56 \pm 3.30$ & $24.018 \pm 3.37$ & $25.03 \pm 3.14^{* *}$ & 0.0001 \\
\hline $\mathrm{FPG}(\mathrm{mmol} / \mathrm{L})$ & $4.87 \pm 0.68$ & $4.97 \pm 0.70$ & $5.18 \pm 0.90^{* *}$ & 0.001 \\
\hline 1hPG (mmol/L) & $9.11 \pm 2.15$ & $9.19 \pm 2.15$ & $9.66 \pm 2.43^{*}$ & 0.048 \\
\hline 2hPG (mmol/L) & $6.99 \pm 1.98$ & $7.29 \pm 2.12$ & $7.64 \pm 2.36^{* *}$ & 0.022 \\
\hline HbAlc (\%) & $5.55 \pm 0.46$ & $5.52 \pm 0.45$ & $5.62 \pm 0.44$ & 0.228 \\
\hline GA (\%) & $13.63 \pm 1.38$ & $13.21 \pm 1.24$ & $13.36 \pm 1.26$ & 0.090 \\
\hline $\mathrm{TC}(\mathrm{mmol} / \mathrm{L})$ & $5.09 \pm 0.99$ & $5.14 \pm 0.97$ & $5.36 \pm 0.91^{*}$ & 0.026 \\
\hline $\mathrm{TG}(\mathrm{mmol} / \mathrm{L})$ & $1.05 \pm 0.57$ & $1.23 \pm 0.82^{*}$ & $1.72 \pm 0.97^{* *}$ & 0.0001 \\
\hline HDL-C (mmol/L) & $1.54 \pm 0.32$ & $1.53 \pm 0.39$ & $1.34 \pm 0.85^{* *}$ & 0.0001 \\
\hline $\mathrm{LDL}-\mathrm{C}(\mathrm{mmol} / \mathrm{L})$ & $3.04 \pm 0.92$ & $3.08 \pm 0.78$ & $3.39 \pm 0.73^{* *}$ & 0.001 \\
\hline HOMA- $\beta$ (\%) & $84.51(55.46-131.82)$ & $88.50(54.81-142.38)$ & $90.95(59.12-154.91)$ & 0.120 \\
\hline HOMA-IR & $1.10(0.69-1.63)$ & $1.21(0.83-1.93)$ & $1.59(0.97-2.43)^{* *}$ & 0.0001 \\
\hline DM family history (\%) & $54(31.58)$ & $44(25.73)$ & $53(30.99)$ & 0.426 \\
\hline Insulin treatment therapy (\%) & $48(28.07)$ & $58(33.92)$ & $65(38.01)$ & 0.147 \\
\hline Postpartum AGM (\%) & $46(26.90)$ & $57(33.33)$ & $74(43.27)^{* *}$ & 0.006 \\
\hline Macrosomia (\%) & $9(5.26)$ & $16(9.36)$ & $9(5.26)$ & 0.213 \\
\hline
\end{tabular}

Data are expressed as the mean \pm SD or median (interquartile range). TG: triglyceride; BMI: body mass index; FPG: fasting plasma glucose; $1 \mathrm{hPG:} 1 \mathrm{~h}$ plasma glucose; 2hPG: 2 h plasma glucose; HbAlc: hemoglobin Alc; GA: glycated albumin; TC: total cholesterol; HDL-C: high-density lipoprotein-cholesterol; LDL-C: low-density lipoprotein-cholesterol; DM: diabetes mellitus; AGM: abnormal glucose metabolism; HOMA-IR: homeostasis model assessment for insulin resistance index; HOMA- $\beta$ : homeostasis model assessment for $\beta$ cell function. ${ }^{*}$ Compared to the low tertile group, $p<0.05$. ${ }^{* *}$ Compared to the low tertile group, $p<0.01$. 
TABLE 3: Elevated TG levels as a biomarker for the development of postpartum AGM in GDM patients.

\begin{tabular}{lcc}
\hline Variables & OR & 95\% CI \\
\hline TG levels at 24-28 gestational weeks (mmol/L) & & \\
Low tertile & Reference & $0.597-2.100$ \\
Intermediate tertile & 1.120 & $\mathbf{1 . 0 5 4 - 3 . 8 0 0}$ \\
High tertile & $\mathbf{2 . 0 0 1}$ & $0.986-1.110$ \\
Age (years) & 1.046 & $0.896-1.072$ \\
Prepregnancy BMI $\left(\mathrm{kg} / \mathrm{m}^{2}\right)$ & 0.980 & $0.550-1.841$ \\
Family history of DM & 1.007 & $0.816-2.611$ \\
Insulin treatment during pregnancy & 1.460 & 0.135 \\
FPG at 24-28 gestational weeks (mmol/L) & 0.852 & $\mathbf{1 . 4 1 5 - 5 . 2 9 9}$ \\
HbA1c at 24-28 gestational weeks (\%) & $\mathbf{2 . 7 3 8}$ & 0.661 \\
Macrosomia & 1.011 & 0.983 \\
ALT at 24-28 gestational weeks (U/L) & 0.985 & 0.202 \\
AST at 24-28 gestational weeks (U/L) & 1.019 & $0.953-1.018$ \\
\hline
\end{tabular}

TG: triglyceride; DM: diabetes mellitus; BMI: body mass index; FPG: fasting plasma glucose; HbA1c: hemoglobin A1c; ALT: alanine aminotransferase; AST: aspartate aminotransferase.

addition to elevated TG levels, we also found that $\mathrm{HbA1c}$ during pregnancy (OR 2.738, 95\% CI 1.415-5.299, $p=$ 0.003 ) was a risk factor for postpartum AGM. However, no association was found between other factors and postpartum AGM.

Then, the TG levels after delivery were also determined. We examined the interaction term between TG levels during pregnancy and the postpartum period, and the result was statistically significant $(p<0.05)$. Therefore, we examined the association between TG levels during the second trimester and the risk of postpartum AGM stratified by TG levels after delivery. Women both in the high TG tertile during pregnancy and with ATG levels postpartum were three times as likely as those in the low tertile with NTG levels postpartum (OR 2.925, 95\% CI 1.271-6.730, $p=0.012$ ) to develop AGM postpartum. Moreover, women in the high tertile with NTG levels postpartum were twice as likely as those in the low tertile with NTG levels postpartum (OR 1.984, 95\% CI 1.022$4.365, p=0.046$ ) to develop AGM postpartum (Figure 2). Therefore, the association with postpartum AGM was strong among women with high TG levels in the second trimester, regardless of whatever TG levels after delivery.

\section{Discussion}

In this study, we found that GDM patients with elevated TG levels during pregnancy not only had more severe glucose and lipid disorders and insulin resistance at the time of the measurement but also were at an increased risk for the development of postpartum AGM.

The incidence of AGM after GDM had been reported to vary from $2.6 \%$ to $38 \%$ within 12 weeks after delivery, which was significantly higher than NGT women during pregnancy [18-20]. Another study showed that approximately $70 \%$ of GDM patients would eventually develop DM [21]. Therefore, it is important to determine the risk factors for progression to T2DM over the long term [22]. A systematic review showed that prepregnancy BMI, family history of diabetes, advanced

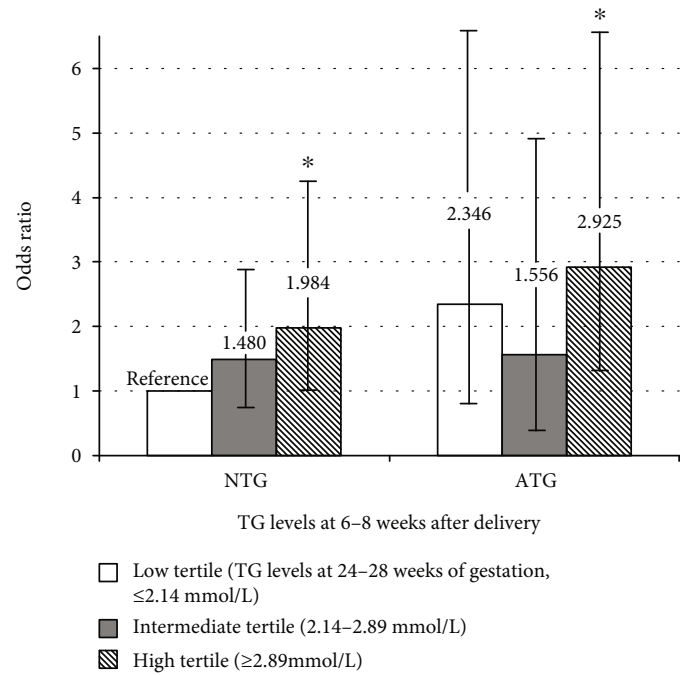

FIGURE 2: Odds ratios and $95 \%$ confidence intervals for postpartum AGM associated with TG levels (the interaction during pregnancy and the postpartum period). AGM: abnormal glucose metabolism; TG: triglyceride; NTG: normal TG levels after delivery $(<1.70 \mathrm{mmol} / \mathrm{L}) ;$ ATG: abnormal TG levels after delivery $(\geq 1.70 \mathrm{mmol} / \mathrm{L})$. ${ }^{*}$ Compared to the reference group, $p<0.05$.

maternal age, increased HbAlc levels, and increased insulin use during pregnancy were associated with the future development of T2DM in GDM patients [23]. During pregnancy, some studies found that high TG levels during gestation were independently associated with an increased risk of GDM $[24,25]$. Furthermore, Kim et al. suggested that postpartum TG was one risk factor for postpartum glucose intolerance [26]. We evaluated the TG levels both during midterm and after delivery to show that TG levels during pregnancy might be an independent biomarker for long-term glucose metabolism in GDM patients, which might be more meaningful compared to TG levels after delivery. Hypertriglyceridemia during pregnancy could not only induce acute 
pancreatitis at the time [27] but also was associated with increased risk of pregnancy-induced hypertension and preterm labor [28, 29]. The midterm elevated TG levels and association with GDM glucose outcome after delivery reminded us to pay more attention to hypertriglyceridemia during pregnancy in GDM patients to prevent postpartum DM.

The serum levels of lipids, including TC, TG, LDL-C, HDL-C, and phospholipids, gradually increase starting in the 12th week of pregnancy and show more pronounced increases during the second and third trimesters [30-32]. Exaggerated TG rises have been found in GDM patients in all trimesters of pregnancy compared to women with NGT [33]. We found that GDM patients with elevated TG levels in the midterm of pregnancy had higher glucose levels and more severe insulin resistance during both pregnancy and the postpartum period. Free fatty acids (FFAs) potentially derived from elevated TGs might decrease insulin sensitivity, creating a vicious cycle between TG levels and insulin resistance $[8,34,35]$. Excess FFAs could result in the generation of toxic lipids, including diacylglycerides and ceramides. These toxic lipids contribute to endoplasmic reticulum stress, mitochondrial dysfunction, and the generation of reactive oxygen species, which together trigger inflammation and insulin resistance [36-39]. FFAs also impact the fatty acid composition of cellular membranes. This directly affects cell function as well as the incorporation of insulin receptors into the membrane $[40,41]$. A higher concentration of TGs during pregnancy leads to subsequent impairment of glucose regulation mainly via FFAs, but further research is needed to explore the detailed mechanism.

This study had some strengths and limitations. This was a study focusing on the association between TG levels and the risk of AGM after delivery, which was a novel perspective. The TG levels were measured at two time points, namely, during pregnancy and the postpartum period, revealing that TG levels during pregnancy were independent factors that affect postpartum glucose metabolism. However, we did not collect information about the lifestyles of our participants, such as dietary factors and physical activity, which could act as confounders. In addition, we did not measure FFA levels, which might be relevant to detailed mechanisms because this measurement was not included in the routine checklist in the clinic. It would be preferable if the TG levels during early and late pregnancy were measured, which would help us to assess the changes in TG levels during different trimesters and the influence of these changes on postpartum glucose metabolism.

In conclusion, our data showed that elevated TG levels during the second trimester were associated with an increased risk of postpartum AGM among women with previous GDM. For those patients, a low-fat diet, lifestyle modifications, and intensified follow-up are strongly recommended to prevent postpartum DM.

\section{Data Availability}

The data used to support the findings of this study are available from the corresponding author upon request.

\section{Conflicts of Interest}

The authors declare that there are no conflicts of interest regarding the publication of this paper.

\section{Authors' Contributions}

Mengyu Lai and Fang Fang contributed equally to this work.

\section{Acknowledgments}

The authors thank all the study participants, research staff, and students who participated in this work. The study was supported by the National Natural Science Foundation of China, Grant/Award Number 81870610; the Shanghai Science and Technology Commission Foundation, Grant/Award Number 18411968800; and the Shanghai "Rising Stars of Medical Talent" Outstanding Youth Medical Talents and the Clinical Research Innovation Plan of Shanghai General Hospital, Grant/Award Number CTCCR-2018C13.

\section{References}

[1] T. Cundy, E. Ackermann, and E. A. Ryan, "Gestational diabetes: new criteria may triple the prevalence but effect on outcomes is unclear," BMJ, vol. 348, no. mar11 6, pp. g1567g1567, 2014.

[2] B. Daly, K. A. Toulis, N. Thomas et al., "Increased risk of ischemic heart disease, hypertension, and type 2 diabetes in women with previous gestational diabetes mellitus, a target group in general practice for preventive interventions: a populationbased cohort study," PLoS Medicine, vol. 15, no. 1, 2018.

[3] L. G. Grunnet, S. Hansen, L. Hjort et al., "Adiposity, dysmetabolic traits, and earlier onset of female puberty in adolescent offspring of women with gestational diabetes mellitus: a clinical study within the Danish National Birth Cohort," Diabetes Care, vol. 40, no. 12, pp. 1746-1755, 2017.

[4] L. Jovanovic, "Gestational diabetes mellitus," JAMA, vol. 286, no. 20, pp. 2516-2518, 2001.

[5] L. Bellamy, J. P. Casas, A. D. Hingorani, and D. Williams, "Type 2 diabetes mellitus after gestational diabetes: a systematic review and meta-analysis," Lancet, vol. 373, no. 9677, pp. 1773-1779, 2009.

[6] R. Retnakaran, Y. Qi, M. Sermer, P. W. Connelly, A. J. Hanley, and B. Zinman, "Glucose intolerance in pregnancy and future risk of pre-diabetes or diabetes," Diabetes Care, vol. 31, no. 10, pp. 2026-2031, 2008.

[7] American Diabetes Association, "Management of diabetes in pregnancy: standards of medical care in diabetes-2020," Diabetes Care, vol. 43, Suppl. 1, pp. S183-S192, 2020.

[8] H. N. Ginsberg, Y. L. Zhang, and A. Hernandez-Ono, "Regulation of plasma triglycerides in insulin resistance and diabetes," Archives of Medical Research, vol. 36, no. 3, pp. 232-240, 2005.

[9] K. Ryckman, C. Spracklen, C. Smith, J. Robinson, and A. Saftlas, "Maternal lipid levels during pregnancy and gestational diabetes: a systematic review and meta-analysis," Bjog An International Journal of Obstetrics \& Gynaecology., vol. 122, no. 5, pp. 643-651, 2015.

[10] E. Herrera, "Lipid metabolism in pregnancy and its consequences in the fetus and newborn," Endocrine, vol. 19, no. 1, pp. $43-55,2002$. 
[11] A. Dotevall, S. Johansson, L. Wilhelmsen, and A. Rosengren, "Increased levels of triglycerides, BMI and blood pressure and low physical activity increase the risk of diabetes in Swedish women. A prospective 18-year follow-up of the BEDA study," Diabetic Medicine, vol. 21, no. 6, pp. 615-622, 2004.

[12] J. B. McPhillips, E. Barrett-Connor, and D. L. Wingard, "Cardiovascular disease risk factors prior to the diagnosis of impaired glucose tolerance and non-insulin-dependent diabetes mellitus in a community of older adults," American Journal of Epidemiology, vol. 131, no. 3, pp. 443-453, 1990.

[13] A. N. Sweeting, J. Wong, H. Appelblom et al., “A novel early pregnancy risk prediction model for gestational diabetes mellitus," Fetal Diagnosis and Therapy., vol. 45, no. 2, pp. 76-84, 2019.

[14] International Association of Diabetes and Pregnancy Study Groups Consensus Panel, "International association of diabetes and pregnancy study groups recommendations on the diagnosis and classification of hyperglycemia in pregnancy," Diabetes Care, vol. 33, pp. 676-682, 2010.

[15] P. G. Colman, D. W. Thomas, P. Z. Zimmet, T. A. Welborn, P. Garcia-Webb, and M. P. Moore, "New classification and criteria for diagnosis of diabetes mellitus. The Australasian Working Party on Diagnostic Criteria for Diabetes Mellitus," The New Zealand Medical Journal, vol. 112, no. 1086, pp. 139-141, 1999.

[16] R. Kahn, J. Buse, E. Ferrannini, and M. Stern, "The metabolic syndrome: time for a critical appraisal. Joint statement from the American Diabetes Association and the European Association for the Study of Diabetes," Diabetologia, vol. 48, no. 9, pp. 1684-1699, 2005.

[17] T. M. Wallace, J. C. Levy, and D. R. Matthews, "Use and abuse of HOMA modeling," Diabetes Care, vol. 27, no. 6, pp. 14871495, 2004.

[18] M. P. Carson, M. I. Frank, and E. Keely, "Original research: postpartum testing rates among women with a history of gestational diabetes-systematic review," Primary Care Diabetes., vol. 7, no. 3, pp. 177-186, 2013.

[19] S. Mcclean, D. Farrar, C. A. Kelly, D. J. Tuffnell, and D. C. Whitelaw, "The importance of postpartum glucose tolerance testing after pregnancies complicated by gestational diabetes," Diabetic Medicine., vol. 27, no. 6, pp. 650-654, 2010.

[20] J. Ogonowski and T. Miazgowski, "The prevalence of 6 weeks postpartum abnormal glucose tolerance in Caucasian women with gestational diabetes," Diabetes Research and Clinical Practice, vol. 84, no. 3, pp. 239-244, 2009.

[21] C. Kim, K. M. Newton, and R. H. Knopp, "Gestational diabetes and the incidence of type 2 diabetes: a systematic review," Diabetes Care, vol. 25, no. 10, pp. 1862-1868, 2002.

[22] L. Leuridan, J. Wens, R. Devlieger, J. Verhaeghe, C. Mathieu, and K. Benhalima, "Glucose intolerance in early postpartum in women with gestational diabetes: who is at increased risk?," Primary Care Diabetes, vol. 9, no. 4, pp. 244-252, 2015.

[23] G. Rayanagoudar, A. A. Hashi, J. Zamora, K. S. Khan, G. A. Hitman, and S. Thangaratinam, "Quantification of the type 2 diabetes risk in women with gestational diabetes: a systematic review and meta-analysis of 95,750 women," Diabetologia, vol. 59, no. 7, pp. 1403-1411, 2016.

[24] W.-Y. Jin, S.-L. Lin, R.-L. Hou et al., "Associations between maternal lipid profile and pregnancy complications and perinatal outcomes: a population-based study from China," $B M C$ Pregnancy and Childbirth, vol. 16, no. 1, 2016.
[25] W. Bao, S. Dar, Y. Zhu et al., "Plasma concentrations of lipids during pregnancy and the risk of gestational diabetes mellitus: a longitudinal study," Journal of Diabetes, vol. 10, no. 6, pp. 487-495, 2018.

[26] S. H. Kim, M. Y. Kim, J. H. Yang et al., "Nutritional risk factors of early development of postpartum prediabetes and diabetes in women with gestational diabetes mellitus," Nutrition, vol. 27, no. 7-8, pp. 782-788, 2011.

[27] N. Ewald, P. D. Hardt, and H. U. Kloer, "Severe hypertriglyceridemia and pancreatitis: presentation and management," Current Opinion in Lipidology., vol. 20, no. 6, pp. 497-504, 2009.

[28] H. Shen, X. Liu, Y. Chen, B. He, and W. Cheng, "Associations of lipid levels during gestation with hypertensive disorders of pregnancy and gestational diabetes mellitus: a prospective longitudinal cohort study," BMJ Open, vol. 6, no. 12, 2016.

[29] L. A. N. A. Y. M. MUDD, C. L. A. U. D. I. A. B. HOLZMAN, J. A. N. E. T. M. CATOV, P. A. T. R. I. C. I. A. K. SENAGORE, and R. H. O. B. E. R. T. W. EVANS, "Maternal lipids at midpregnancy and the risk of preterm delivery," Acta Obstetricia et Gynecologica Scandinavica, vol. 91, no. 6, pp. 726-735, 2012.

[30] Ä. Bartels, N. Egan, D. I. Broadhurst et al., "Maternal serum cholesterol levels are elevated from the 1st trimester of pregnancy: a cross-sectional study," Journal of Obstetrics and Gynaecology, vol. 32, no. 8, pp. 747-752, 2012.

[31] J. Villar, L. Cheikh Ismail, C. G. Victora et al., "International standards for newborn weight, length, and head circumference by gestational age and sex: the newborn cross-sectional study of the INTERGROWTH-21st project," Lancet, vol. 384, no. 9946, pp. 857-868, 2014.

[32] A. Ghio, A. Bertolotto, V. Resi, L. Volpe, and G. Di Cianni, "Triglyceride metabolism in pregnancy," Advances in Clinical Chemistry, vol. 55, pp. 133-153, 2011.

[33] E. Herrera and H. Ortega-Senovilla, "Disturbances in lipid metabolism in diabetic pregnancy-are these the cause of the problem?," Best Practice \& Research. Clinical Endocrinology \& Metabolism, vol. 24, no. 4, pp. 515-525, 2010.

[34] M. M. Lasram, K. Bouzid, I. B. Douib et al., "Lipid metabolism disturbances contribute to insulin resistance and decrease insulin sensitivity by malathion exposure in Wistar rat," Drug and Chemical Toxicology, vol. 38, no. 2, pp. 227-234, 2014.

[35] A. Kotronen, V. R. Velagapudi, L. Yetukuri et al., "Serum saturated fatty acids containing triacylglycerols are better markers of insulin resistance than total serum triacylglycerol concentrations," Diabetologia, vol. 52, no. 4, pp. 684-690, 2009.

[36] B. Sears and M. Perry, "The role of fatty acids in insulin resistance," Lipids in Health and Disease, vol. 14, no. 1, 2015.

[37] N. Lin, H. Chen, H. Zhang, X. Wan, and Q. Su, "Mitochondrial reactive oxygen species (ROS) inhibition ameliorates palmitate-induced INS-1 beta cell death," Endocrine, vol. 42, no. 1, pp. 107-117, 2012.

[38] Y. S. Oh, G. D. Bae, D. J. Baek, E.-Y. Park, and H.-S. Jun, "Fatty acid-induced lipotoxicity in pancreatic beta-cells during development of type 2 diabetes," Frontiers in Endocrinology, vol. 9, 2018.

[39] K. L. Fritsche, "The science of fatty acids and inflammation," Advances in Nutrition, vol. 6, no. 3, pp. 293S-301S, 2015.

[40] J. Miyamoto, M. Kasubuchi, A. Nakajima, and I. Kimura, "Antiinflammatory and insulin-sensitizing effects of free fatty acid receptors," Free Fatty Acid Receptors, vol. 236, p. 221, 2016.

[41] M. Pilon, "Revisiting the membrane-centric view of diabetes," Lipids in Health and Disease., vol. 15, no. 1, p. 167, 2016. 